\section{THU0507 LONG-TERM EXPERIENCE WITH ADALIMUMAB FOR THE TREATMENT OF JUVENILE IDIOPATHIC ARTHRITIS. 5- YEAR DATA FROM THE GERMAN BIKER REGISTRY}

A. Klein ${ }^{1}$, K. Minden ${ }^{2}$, I. Foeldvari ${ }^{3}$, G. Ganser ${ }^{4}$, J.P. Haas ${ }^{5}$, R. Trauzeddel ${ }^{6}$ A. Hospach ${ }^{7}$, F. Weller-Heinemann ${ }^{8}$, G. Horneff ${ }^{1}$ on behalf of BIKER collaborative group. ${ }^{1}$ Asklepios, Sankt Augustin; ${ }^{2}$ Charité, Berlin; ${ }^{3}$ Private office, Hamburg; ${ }^{4}$ Ped Rheumatol, Sendenhorst; ${ }^{5}$ German Ped Rheum Centre, Garmisch-Partenkirchen; ${ }^{6}$ Chidrens Hospital, Helios Klinikum Berlin Buch, Berlin; ${ }^{7}$ Paediatric Rheumatology, Olgahospital, Stuttgart; ${ }^{8}$ Paediatric Rheumatology, Klinikum Bremen Mitte, Bremen, Germany

Background: Since the approval of Adalimumab (ADA) for treatment of juvenile idiopathic arthritis (JIA), it has become a valuable option, which significantly improved the outcome of patients.

Objectives: To report efficacy (as observed) and safety of ADA in clinical practice. Methods: Data from the German BiKeR register from 2011 to 2016 are reported. Baseline patient characteristics, treatment response and safety data were compared. Treatment response was analyzed using JIA-ACR criteria, JADAS score and improvement of functional status (Childhood Health Assessment Questionnaire disability index, CHAQ), JIA-ACR-scores, JADAS10-minimal disease activity (MDA), JADAS-remission and ACR-inactive disease criteria were analysed.

Results: 589 non-systemic JIA patients exposed to Adalimumab with at least one follow-up report were identified in the German BIKER registry, representing 1143.9 patient years (PY) of exposure to ADA and 1206.5 observation years including 90 days after discontinuation. At Baseline, 58.2\% received combination with methotrexate (MTX). Patients on combination treatment had more frequently ANA, less frequently HLA-B27, had higher JADAS 10 and more often received systemic steroids $(9.9 \%$ vs. $22.2 \%, p=0.0002)$. ADA dosage was $0.8 \mathrm{mg} / \mathrm{kg}$ in both cohorts.

At month 12 JIA-ACR 30/50/70/90 and JADAS-MDA/-remission/ACR-inactive disease was reached by $66 / 63 / 49 / 33 / 50 / 24 / 27 \%$ on monotherapy with ADA and $68 / 63 / 45 / 28 \% / 50 / 28 / 27$ on combination of ADA and MTX (not significant). Response rates at month 24 were $67 / 66 / 54 / 35 / 65 / 32 / 29 \%$ on ADA monotherapy and $67 / 61 / 46 / 31 / 57 / 35 / 30 \%$ on ADA+MTX combination cohort (not significant). The rate (/100PY) of all adverse events (AE)/serious AE/infections/serious infections/uveitis events was 52.3/2.2/16.4/4.6 upon monotherapy and 65.3/5.1/2.3/16.4/6.2 upon combination. Only rates for any AE were significantly higher upon combination $(\mathrm{p}=0.008)$ as well as rate of elevated transaminase $(p=0.01)$. Rate of patients with an uveitis event was higher upon combination ( $9.6 \%$ vs. $5.3 \%, p=0.007)$

Table 1

\begin{tabular}{|c|c|c|c|}
\hline & ADA monotherapy & $A D A+M T X$ & $\mathrm{P}$ \\
\hline $\mathrm{N}$, female $(\%)$ & $223(70)$ & $343(70)$ & n.s. \\
\hline Age onset/disease duration; mean (SD) & $7.1( \pm 4.3) / 6.0(3.7)$ & $6.5(4.5) / 5.0(3.7)$ & n.s. \\
\hline RF neg. Poylarthritis, $n(\%)$ & $77(34.5)$ & $126(36.7)$ & n.s. \\
\hline RF pos. Poylarthritis, $n(\%)$ & $7(3.1)$ & $17(5.0)$ & n.s. \\
\hline pers. Oligoarthritis, $n(\%)$ & $12(5.4)$ & $29(8.5)$ & n.s. \\
\hline ext. Oligoarthritis, n (\%) & $59(26.5)$ & $95(27.7)$ & n.s. \\
\hline ERA, $n(\%)$ & $49(22.0)$ & $47(13.7)$ & $\mathrm{p}=0.01$ \\
\hline PsA, n (\%) & $15(6.7)$ & $22(6.4)$ & n.s. \\
\hline unclassified JIA, n (\%) & $4(1.8)$ & $7(2.0)$ & n.s. \\
\hline \multirow[t]{2}{*}{ ANA/B27, n (\%) } & $111(49.8) /$ & $210(61.2) /$ & $\mathrm{p}=0.009$ \\
\hline & $56(25.1)$ & $57(16.6)$ & $p=0.02$ \\
\hline Comorbidity Uveitis, $n(\%)$ & $19(8.5)$ & $32((9.3)$ & n.s. \\
\hline Baseline active joints mean (SD) & $3.5(5.6)$ & $3.8(5.5)$ & n.s. \\
\hline Phy VAS mean (SD) & $37.6(28.6)$ & $42.7(28.3)$ & n.s. \\
\hline Pat VAS mean (SD) & $27.9(24.9)$ & $30.9(24.9)$ & n.s. \\
\hline $\mathrm{CHAQ}$ mean $(\mathrm{SD})$ & $0.4(0.6)$ & $0.4(0.6)$ & n.s. \\
\hline ESR mean (SD) & $14.3(15.2)$ & $18.1(17.7)$ & n.s. \\
\hline CRP mean (SD) & $4.3(7.4)$ & $8.3(15.8)$ & n.s. \\
\hline Baseline JADAS10 mean (SD) & $9.9(7.0)$ & $11.3(7.1)$ & $\mathrm{p}=0.02$ \\
\hline
\end{tabular}

Efficacy parameters after 12 month of ADA treatment

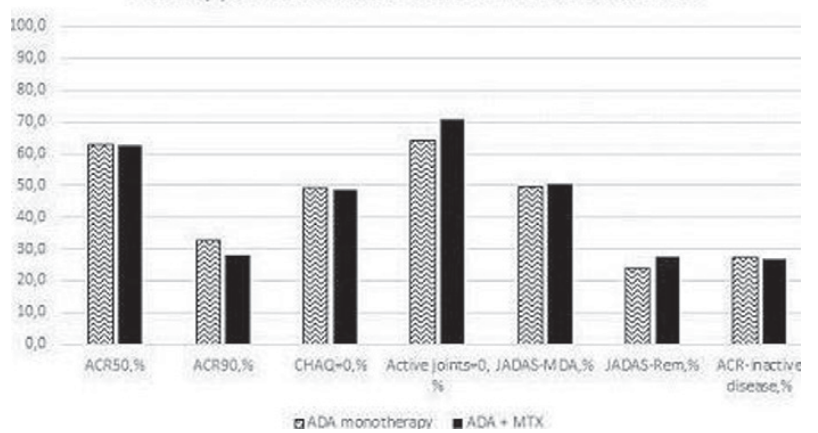

Conclusions: Adalimumab demonstrated high response rates and an acceptable risk profile. Efficacy and safety of monotherapy was not inferior to combination therapy with MTX.

Disclosure of Interest: A. Klein: None declared, K. Minden Speakers bureau: Abbvie, Pfizer,Roche,Genzyme,Pharm-Allergan, I. Foeldvari: None declared, G. Ganser: None declared, J. Haas: None declared, R. Trauzeddel: None declared,
A. Hospach: None declared, F. Weller-Heinemann: None declared, G. Horneff Grant/research support from: Abbvie, Chugai, Novartis, Pfizer,Roche DOI: 10.1136/annrheumdis-2017-eular.3457

\section{THU0508 PSYCHOLOGICAL FEATURES OF CHILDREN WITH RHEUMATIC DISEASES}

I. Chyzheuskaya ${ }^{1}$, L. Byelyaeva ${ }^{1}$, R. Filonovich ${ }^{2}$, L. Zajtseva ${ }^{2}{ }^{1}$ Pediatrics, Belarusian Medical Academy of Postgraduate Education; ${ }^{2}$ Pediatrics, 4th City Children's Clinical Hospital of Minsk, Minsk, Belarus

Background: Rheumatic diseases (RD) in children are characterized by a wide spectrum of clinical manifestations, and variability of flow, the defeat of many body systems, including the musculoskeletal system, which often leads to early disability. The disease brings the children not only physical suffering and psychological distress as a result of the pathological process may be sufficiently stable defects in appearance, affecting the minds of the growing person.

Objectives: To study the psychological characteristics of children with rheumatic diseases.

Methods: 167 patients with RD were examined in the period from 2008 to 2015. In the I group included 115 children with juvenile idiopathic arthritis (JIA) (mean age $11,9 \pm 3,4$ years), II group consisted of 34 children with juvenile scleroderma (JS) (mean age 12,4 $\pm 2,8$ years), III - 18 children with systemic lupus erythematosus (SLE) (mean age 13,1+1,7 years). As control group examined 30 healthy children aged 9-17 years (mean age 12,93 $\pm 0,4$ years). To investigate the expression of reactive anxiety $(\mathrm{RA})$ and personal anxiety $(\mathrm{PA})$ in children older than 9 years was used scale Ch.D.Spielberg. In order to study the intensity of anxiety was used M.Luscher color test. To identify the individual psychological characteristics of the individual patient questionnaire used G.J.Eysenck for teenagers, consisting of 60 questions, including the scale of extroversion, introversion, neuroticism and the scale of "the scale of lies". Testing patients and interpretation of the results was carried out together with a psychologist.

Results: The test Spielberg results indicate that significantly more $(P<0.001)$, medium and high levels of anxiety both reactive and personal, occurred in children with RD. This suggests the presence of children with RD border states with the threat of self-assessment features, reducing the threshold of resistance to stressful situations. The average RA and PA indices were significantly higher $(P<0.001)$ in children with RD in comparison with indicators of reactive and personal anxiety in children in the control group $(46,09 \pm 0,88$ points for the JS, $43,77 \pm 1,37$ points for $\mathrm{JIA}, 45,07 \pm 1,83$ points for SLE and $27,6 \pm 0,62$ points for the control group, respectively, RA; $42,22+1,68$ points for the JS, 37,84 $\pm 1,27$ points for the JIA, 44,76 $\pm 0,88$ points for SLE and $28,7 \pm 0,51$ points, respectively, RA for the control group).

Results of the study with the help of M.Lusher's color test revealed the intensity of anxiety in children with JS, SLE and JIA. Evaluation results of the study showed that children and adolescents with RD clearly manifested trend bias pri-mary colors on the $6,7,8$ positions, and more - in the first place.

In order to identify characteristics of temperament in patients aged 13-17 years were asked to complete a questionnaire G.J.Eysenck for undergrowth Cove. The majority of surveyed teens both clinical groups manifest emotional instability (73.3\% of patients with JS and $70 \%$ of patients with $\mathrm{JIA}$ ) and features typical of introversion $(60 \%$ of patients with SS and $68 \%$ of patients with JIA) (verified differences were found) between the groups. According to G.J.Eysenck, high rates of introversion in conjunction with emotional instability correspond to the alarm state.

Conclusions: As a result, psychometric studies found that the characteristic of emotional tension, anxiety, isolation for children with RD.

Disclosure of Interest: None declared

DOI: 10.1136/annrheumdis-2017-eular.6869

\section{THU0509 IMPROVEMENT OF DISEASE ACTIVITY IN PATIENTS WITH COLCHICINE-RESISTANT FMF, HIDS/MKD AND TRAPS ASSESSED BY AUTOINFLAMMATORY DISEASE ACTIVITY INDEX (AIDAI): RESULTS FROM THE CLUSTER TRIAL}

I. Kone-Paut ${ }^{1}$, M. Piram ${ }^{1}$, S. Benseler ${ }^{2}$, M. Hofer ${ }^{3}$, H. Lachmann ${ }^{4}$, H.M. Hoffman ${ }^{5}$, M. Gattorno ${ }^{6}$, J. Frenkel ${ }^{7}$, J.B. Kuemmerle-Deschner ${ }^{8}$, S. Ozen ${ }^{9}$, J. Levy ${ }^{10}$, C.S. Karyekar ${ }^{11}$, F. De Benedetti ${ }^{12} .{ }^{1}$ APHP, CHU de Bicêtre, Univ Paris Sud, le Kremlin Bicêtre, France; ${ }^{2}$ Alberta Children's Hospital, Calgary, Canada: ${ }^{3}$ University of Lausanne, Lausanne, Switzerland: ${ }^{4}$ UK National Amyloidosis Centre, London, United Kingdom: ${ }^{5}$ University of California, la Jolla, United States; ${ }^{6}$ G. Gaslini Institute, Genoa, Italy; ${ }^{7}$ University Medical Center, Utrecht, Netherlands; ${ }^{8}$ University Hospital Tuebingen, Tuebingen, Germany; ${ }^{9}$ Hacettepe University, Ankara, Turkey; ${ }^{10}$ BIOP, Reinach, Switzerland; ${ }^{11}$ Novartis Pharmaceuticals Corporation, East Hanover, United States; ${ }^{12}$ IRCCS Ospedale Pediatrico Bambino Gesú, Rome, Italy

Background: AIDAI is a novel validated tool for assessment of disease activity in autoinflammatory diseases. ${ }^{1}$ CLUSTER study (NCT02059291) demonstrated that canakinumab (CAN; an anti- IL-1 $\beta$ antibody) is efficacious in resolving active flare and in preventing new flare in patients (pts) with colchicine resistant familial Mediterranean fever (crFMF), hyper-IgD syndrome/mevalonate kinase deficiency (HIDS/MKD) and TNF receptor-associated periodic syndrome (TRAPS). ${ }^{2}$ 